\title{
Quantitative readout of optically-encoded gold nanorods using an ordinary dark-field microscope
}

\author{
Raffaella Mercatelli, ${ }^{a}$ Fulvio Ratto, ${ }^{b}{ }^{b}$ Sonia Centi, ${ }^{c}$ Silvia Soria, ${ }^{b}$ Giovanni Romano, ${ }^{b}$ Paolo \\ Matteini, ${ }^{b}$ Franco Quercioli, ${ }^{a}$ Roberto Pini, ${ }^{b}$ and Franco Fusi ${ }^{c}$ \\ ${ }^{a}$ INO-CNR, National Institute of Optics-CNR, Largo E. Fermi 6, Florence, Italy. Fax: 0039055 23081; Phone: 00390552337755 \\ ${ }^{b}$ IFAC-CNR, Institute of Applied Physics-CNR, Via Madonna del Piano 10, Sesto Fiorentino, Firenze, Italy. Fax: 0039055 5226477; Phone: \\ 0039055 5225304; E-mail:f.ratto@ifac.cnr.it \\ ${ }^{c}$ Dept Biomedical, Experimental and Clinical Sciences, University of Florence, Viale Pieraccini 6, Florence, Italy. Fax: 00390554271413 ; \\ Phone: 0039055 4271217;
}

\section{ADDITIONAL DETAIL ON THE EXPERIMENTAL APPARATUS AND SAMPLE PREPARATION}

Figure S1 displays a diagram of the optical system that was used to pattern hybrid films of polyvinyl alcohol (PVA) and gold nanoparticles as well as to perform the dark-field microscopy analysis. This setup allows us to simultaneously modify and acquire the multiphoton luminescence from our samples (see e.g. ref [7]), which provides an immediate check of the recording process. In turn, this is used to set suitable recording parameters, including focus, light intensity, pixel dwell time and pulse repetition rate. Briefly, a mode-locked Ti:Sapphire laser (Ti:Sa: Mira 900 F, Coherent, Inc, USA) pumped by a solid-state laser (V5: Verdi, Coherent, Inc, USA) is coupled to a cavity dumper (CD: Pulse Switch for Mira, Coherent, Inc, USA), in order to decrease the original repetition rate of the Mira 900 source (76 MHz). The light is directed through a mirror $(\mathbf{M})$ to a lens $(\mathbf{L})$, which focuses the beam into the Nikon PCM2000 confocal laser scanning unit attached to a TE2000-U inverted optical microscope (CLSM). The 
luminescence of the gold nanoparticles is collected and directed through an output fiber to a photomultiplier tube (PMT) and then visualized on a pc screen.

In this way, it becomes possible to inspect parameters such as the distance among lines, which depends on the laser repetition rate and pixel dwell time, and the extent of damage, which is governed by the focus and light intensity. For instance, an optimal recording is associated with a substantial attenuation of the multiphoton luminescence of the gold nanoparticles, which is attributed to their reshaping decoupling the plasmonic resonances from the incident and emitted light (see e.g. ref 51). However, an excessive overexposure may decompose the PVA scaffold, which enhances its luminescence signals.

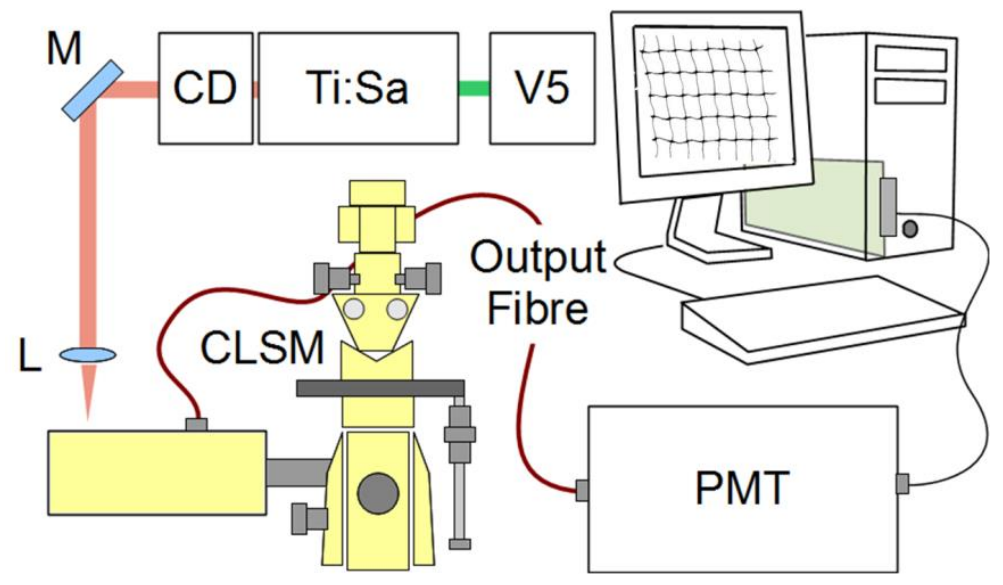

Figure S1: Schematic representation of the setup used to pattern and inspect the hybrid sols of gold nanoparticles and PVA.

Figure S2 displays a dark-field micrograph of a specimen that was prepared by deposition and patterning of a PVA film containing gold nanoparticles on a TEM carbon / formvar / copper grid with mesh pitch of $125 \mu \mathrm{m}$. We note that this image was realized by collecting visible light with high sensitivity in the green window. The film coats the TEM grid with good homogeneity through its bars and holes, which we ascribe to its high hydrophilicity. Any asperity, defect and 
speckle of dust in this multilayered superstructure gives a bright spot overlapping the regular array of edges from the copper substrate. However the $\sim(150 \times 50) \mu \mathrm{m}^{2}$ pattern realized by ultrafast laser excitation is discernible as a dense constellation of bright dots that are aligned in a fishing net. These dots originate from the kind of plastic deformation of the PVA scaffold and gold nanoparticles that is described in the main text, and were used as markers to locate our region of interest.

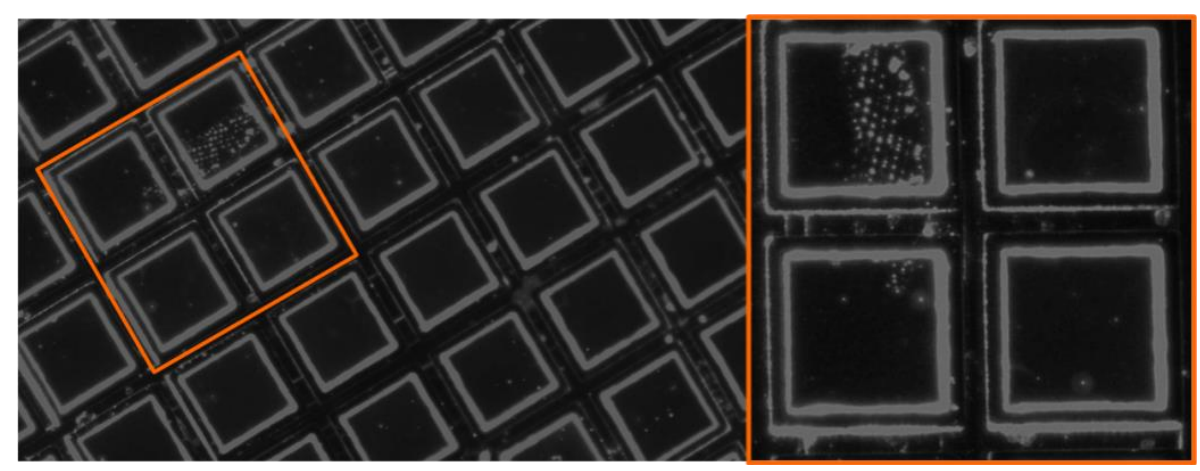

Figure S2: Typical visible dark-field micrograph of a sample prepared on a TEM grid with mesh pitch of $125 \mu \mathrm{m}$. The inset shows a magnification of the square marked with orange edges, and draws attention to the pattern etched by means of the ultrafast laser excitation.

We note that these samples were stable over at least six months at room temperature. The storage was realized under methanol, in order to prevent moisture from swelling the PVA scaffold. 